\title{
Interstitial tumor-associated macrophages combined with tumor-derived colony-stimulating factor-1 and interleukin-6, a novel prognostic biomarker in non-small cell lung cancer
}

\author{
Bao-xiang Pei, MD, ${ }^{\text {a }}$ Bing-sheng Sun, MD, PhD, ${ }^{a}$ Zhen-fa Zhang, MD, PhD, ${ }^{a}$ \\ An-lei Wang, $\mathrm{MD},{ }^{\mathrm{a}}$ and Peng Ren, $\mathrm{MD}, \mathrm{PhD}^{\mathrm{b}}$
}

\begin{abstract}
Objectives: Recent experimental evidence has indicated that interstitial tumor-associated macrophages (TAMs), tumor-derived macrophage colony-stimulating factor (also known as CSF-1), and interleukin-6 (IL-6) interact in the pathogenesis of malignant epithelial tumors, including lung cancer. The present study aimed to explore their relationship and prognostic significance in surgically resected non-small cell lung cancer (NSCLC).
\end{abstract}

\begin{abstract}
Methods: Tissue microarray and immunohistochemistry were used to detect the expression of CSF-1, IL-6, and CD68-positive TAMs in 417 patients with NSCLC undergoing complete pulmonary resection from 2003 to 2008. Their correlations and clinicopathologic data were analyzed using chi-square testing. Their prognostic values were evaluated by univariate Kaplan-Meier survival analysis and multivariate Cox proportional hazard model analysis.
\end{abstract}

Results: The expression of CSF-1 and IL-6 in NSCLC correlated positively with the infiltration degree of TAMs in the tumor stroma ( $r=0.184$ and $r=0.196$, respectively; $P<.001)$. The expression of both CSF-1 and IL-6 was statistically significant for survival $(P<.001)$. Nevertheless, no such relationship was observed for CD68 in the tumor stroma $(P>.05)$. When CSF-1 and/or IL-6 and CD68 were taken into consideration together, the result became statistically significant. Multivariate analysis showed that co-expression of CD68, CSF-1, and IL-6 remained the most significant and independent prognostic factor for survival $(P<.05)$ but not the combinations of CSF-1 and IL-6, CD68 and CSF-1, or CD68 and IL-6 $(P>.05)$. The 5-year survival rate in the CD68-negative and CSF-1- and IL-6-positive group was better than the rate in the CD68, CSF-1-, and IL-6-positive group $(P<.05)$.

Conclusions: The combination of CD68 plus TAMs, CSF-1, and IL-6 is very likely to be a valuable independent predictor of survival in patients with NSCLC. Perhaps co-expression of CSF-1 and IL-6 induces interstitial TAMs to shift toward the tumor-promoting phenotype. (J Thorac Cardiovasc Surg 2014;148:1208-16)

Supplemental material is available online.

\footnotetext{
From the Departments of Lung Cancer ${ }^{\mathrm{a}}$ and Esophageal Cancer, ${ }^{\mathrm{b}}$ Tianjin Medical University Cancer Institute and Hospital, Tianjin Lung Cancer Center, National Clinical Research Center of Cancer, Key Laboratory of Cancer Prevention and Therapy, Tianjin, China.

The present study was funded by the National Natural Science Foundation of China (grant 81201649) and the Tianjin Natural Science Foundation of China (grants 11JCYBJC13300, 12JCYBJC17800, and 12ZCDZSY15400).

Disclosures: Authors have nothing to disclose with regard to commercial support.

Drs B.-x.P and B.-s.S contributed equally to the present report.

Received for publication Feb 26, 2014; revisions received April 21, 2014; accepted for publication May 2, 2014; available ahead of print June 4, 2014.

Address for reprints: Zhen-fa Zhang, MD, PhD, Department of Lung Cancer, Tianjin Medical University Cancer Institute and Hospital, Tianjin Lung Cancer Center, National Clinical Research Center of Cancer, Key Laboratory of Cancer Prevention and Therapy, Huanhu West Rd, Tianjin 300060, China (E-mail: peibaoxiangok@ yahoo.com)

$0022-5223 / \$ 36.00$

Copyright (c) 2014 by The American Association for Thoracic Surgery

http://dx.doi.org/10.1016/j.jtcvs.2014.05.003
}

Lung cancer is the leading cause of cancer mortality in the world, with an overall 5-year relative survival rate of approximately $15 \%$ at diagnosis. ${ }^{1,2}$ Even for patients with pathologic stage IA non-small cell lung cancer (NSCLC), the 5-year overall survival has been approximately $67 \%$. $^{3}$ Approximately $40 \%$ of patients with stage I and those with $60 \%$ of stage II NSCLC will die within 5 years after curative pulmonary resection, mainly because of distant metastases and local recurrence. ${ }^{4-6}$ Because of its prevalence and poor prognosis, the selection of appropriate biomarkers to evaluate severity, monitor progression, and estimate the efficacy of a specific therapy has become an unavoidable issue.

Solid tumors comprise not only cancer cells, but also many other nonmalignant stromal cells, producing a unique microenvironment to modify the neoplastic properties. One such stromal cell type that might promote cancer progression is tumor-associated macrophages (TAMs), which derive from circulating monocytic precursors. ${ }^{7}$ They are recruited to the tumor site by cytokines and other tumor-derived factors 


$$
\begin{array}{ll}
\text { Abbreviations and Acronyms } \\
+ & =\text { positive } \\
- & =\text { negative } \\
\text { CSF-1 } & =\text { colony-stimulating factor- } 1 \\
\text { DFS } & =\text { disease-free survival } \\
\text { IL-6 } & =\text { interleukin- } 6 \\
\text { NSCLC } & =\text { non-small cell lung cancer } \\
\text { OS } & =\text { overall survival } \\
\text { TAM } & =\text { tumor-associated macrophage } \\
\text { TMA } & =\text { tissue microarray }
\end{array}
$$

and, once in situ, produce chemokines and growth and angiogenic factors that alter tumor growth, invasion, and metastasis. ${ }^{8}$ Although an increasing body of preclinical and clinical evidence has associated TAMs with a poor prognosis, their prognostic significance in NSCLC has been contradictory. ${ }^{9-12}$ Therefore, the cytokine profile of the tumor microenvironment is thought to educate TAMs toward a tumor-promoting or tumor-suppressing phenotype; however, the specific mechanisms remain not fully identified.

Several recent studies of ovarian cancer have demonstrated that the tumor-promoting TAMs were induced by interleukin-6 (IL-6), leukemia inhibitory factor, and colony-stimulating factor-1 (CSF-1) derived from cancer cells. $^{13-15}$ The potential relationship and biologic significance between TAMs and tumor-derived CSF-1 and IL-6 have never been clarified in NSCLC. Studies have shown that the pre- and postoperative serum levels of CSF-1 and IL-6 are important prognostic indicators in patients with NSCLC. ${ }^{16,17}$ However, because the serum concentrations can be influenced by many factors, certain limitations exist. The present study intended to directly detect the expression of CSF-1, IL-6, and TAMs in the NSCLC microenvironment and explore their prognostic value. This could be of great significance to further clarify the biologic function of TAMs and thereby open up a new avenue for the comprehensive treatment of NSCLC.

\section{METHODS}

\section{Tissue Samples}

All patients with stage I to IIIA NSCLC undergoing complete pulmonary resection and systematic lymph node dissection at the Cancer Institute and Hospital of Tianjin Medical University (China) from 2003 to 2008 were considered eligible for retrospective analysis of the clinical prognostic factors. Patients were excluded from the present study if they had met any 1 of the following criteria: (1) the use of neoadjuvant therapy; (2) the presence of metastatic disease preoperatively; (3) complete clinicopathologic and follow-up data were not available; (4) death had occurred within 1 month after surgery. The histologic type for all NSCLC cases was re-assessed according to the modern classification system (World Health Organization 2011) by 2 dedicated pathologists. Tumor staging was done using the most recent International Association for the Study of Lung Cancer TNM classification system. ${ }^{18}$ All patients were followed up until September 1, 2013. The research ethics committee of Tianjin Cancer Institute and Hospital provided ethical approval for the study of human subjects, and all patients provided written informed consent. The patients who were still alive after the last follow-up visit and those who had been lost to follow-up were censored in the present study.

\section{Tissue Microarray and Immunohistochemistry}

For tissue microarray (TMA) construction, 2 experienced pathologists reviewed hematoxylin and eosin-stained sections from each paraffinembedded, formalin-fixed block. The most representative areas of the tumor region were carefully selected and sampled for the TMA collector blocks. To validate the concordance between the TMAs and whole tumor sections, we also detected CSF-1, IL-6, and CD68 expression for 40 cases randomly chosen from 417 patients.

Immunohistochemistry was performed with monoclonal anti-mouse CD68 antibody at a 1:5 dilution (clone KP1, Abcam, Cambridge, UK), anti-rabbit CSF-1 polyclonal antibody at a 1:100 dilution (code BA0750, Boster Biological Technology, Wuhan, China), and anti-IL6 rabbit polyclonal antibody at a 1:100 dilution (code BS0781R, Bioss Biological Technology, Beijing, China). After deparaffinization, rehydration, and heat-induced antigen retrieval, the tissues were immersed in methanol containing 3\% hydrogen peroxide for 30 minutes to block the endogenous peroxidase activities. After incubated with normal goat serum (diluted 1:10) for 20 minutes to block the nonspecific antibody-binding sites, the sections were incubated with primary antibodies overnight at $4^{\circ} \mathrm{C}$. After 30 minutes of incubation with the secondary antibody, the sections were developed in diaminobenzidine solution under microscopic observation and counterstained with hematoxylin to visualize the nuclei. Negative control slides with the primary antibodies omitted were included in all assays.

Two pathologists independently evaluated the staining of all anonymized samples. Five different areas at $400 \times$ magnification from each sample were systematically evaluated for the expression of CSF-1, IL-6, and CD68. The mean value of the 5 cores was considered representative of 1 tumor. For CSF-1 and IL-6 staining, we calculated the sum of both the staining intensity ( 0 , negative; 1 , weak; 2 , intermediate; 3 , strong) and the percentage of positive cells $(0$, none or $<5 \%, 1,5 \%$ to $25 \% ; 2,25 \%$ to $50 \% ; 3,>50 \%$ ). Scores of 0 to 2 were regarded as negative and scores of 3 to 6 as positive. For CD68 staining, the tissue samples were divided into 2 groups: negative, no infiltration; and positive, infiltration.

\section{Statistical Analysis}

All statistical analyses were performed using the Statistical Package for Social Sciences, version 16.0 (SPSS, Chicago, Ill), statistical programs. The nonparametric Spearman test was used for correlation analysis; the association between the ranked data and the clinical parameter was analyzed using the chi-square test or Fisher's exact test. Overall survival (OS) was defined as the interval between surgery and death or the last observation. Disease-free survival (DFS) was measured from the date of resection to the detection of recurrent tumor or the last follow-up assessment. OS and DFS were analyzed using the Kaplan-Meier method and the log-rank test, and multivariate analyses were tested using the Cox proportional hazard model.

\section{RESULTS \\ Patient Characteristics}

Of the eligible patients, 3 were excluded because of the use of neoadjuvant therapy and 2 had died within 1 month after surgery, leaving 417 cases for analysis. The mean follow-up period was 43 months (range, 2 to 120). The clinical and histopathologic details of the 417 cases are listed in Tables 1 and 2 . 
TABLE 1. Relationship between CSF-1, IL-6, and CD68 and clinicopathologic factors of patients

\begin{tabular}{|c|c|c|c|c|c|c|c|c|c|}
\hline \multirow[b]{2}{*}{ Clinicopathologic parameter } & \multicolumn{3}{|c|}{ CSF-1 } & \multicolumn{3}{|c|}{ IL-6 } & \multicolumn{3}{|c|}{ CD68 } \\
\hline & Negative & Positive & $P$ value & Negative & Positive & $P$ value & Negative & Positive & $P$ value \\
\hline Gender & & & .997 & & & .098 & & & .144 \\
\hline Male & 108 & 123 & & 81 & 150 & & 39 & 192 & \\
\hline Female & 87 & 99 & & 80 & 106 & & 42 & 144 & \\
\hline Age group & & & .908 & & & .622 & & & .542 \\
\hline$>60 y$ & 89 & 109 & & 74 & 124 & & 36 & 162 & \\
\hline$\leq 60 \mathrm{y}$ & 103 & 116 & & 87 & 132 & & 45 & 174 & \\
\hline Smoking status & & & .084 & & & .127 & & & .768 \\
\hline$\leq 40$ pack-y & 108 & 112 & & 95 & 125 & & 42 & 178 & \\
\hline$>40$ pack-y & 87 & 110 & & 66 & 131 & & 39 & 158 & \\
\hline Resection type & & & .162 & & & .253 & & & .525 \\
\hline Pneumonectomy & 16 & 22 & & 10 & 28 & & 9 & 29 & \\
\hline Lobectomy & 159 & 188 & & 139 & 208 & & 64 & 283 & \\
\hline Bronchial sleeve resection & 20 & 12 & & 12 & 20 & & 8 & 24 & \\
\hline Histologic subtype & & & .882 & & & .078 & & & .530 \\
\hline SCC & 47 & 55 & & 32 & 70 & & 23 & 79 & \\
\hline Adenocarcinoma & 147 & 165 & & 129 & 183 & & 57 & 255 & \\
\hline $\mathrm{AdSq}$ & 1 & 2 & & 0 & 3 & & 1 & 2 & \\
\hline Subcarinal lymph node status & & & .028 & & & $<.001$ & & & .073 \\
\hline Negative & 166 & 170 & & 144 & 192 & & 71 & 265 & \\
\hline Positive & 29 & 52 & & 17 & 64 & & 10 & 71 & \\
\hline TNM stage & & & .030 & & & .010 & & & .507 \\
\hline I & 87 & 75 & & 76 & 86 & & 34 & 128 & \\
\hline II & 49 & 54 & & 39 & 64 & & 22 & 81 & \\
\hline III & 59 & 93 & & 46 & 106 & & 25 & 127 & \\
\hline Involved nodes (n) & & & .020 & & & .017 & & & .701 \\
\hline$<4$ & 154 & 153 & & 129 & 178 & & 61 & 246 & \\
\hline$\geq 4$ & 41 & 69 & & 32 & 78 & & 20 & 90 & \\
\hline Involved nodal stations (n) & & & .004 & & & .010 & & & .333 \\
\hline$\leq 1$ & 147 & 138 & & 122 & 163 & & 59 & 226 & \\
\hline$>1$ & 48 & 84 & & 39 & 93 & & 22 & 110 & \\
\hline Adjuvant chemotherapy & & & .979 & & & .10 & & & .271 \\
\hline Yes & 128 & 146 & & 99 & 175 & & 49 & 225 & \\
\hline No & 67 & 76 & & 62 & 81 & & 32 & 111 & \\
\hline Adjuvant radiotherapy & & & .770 & & & .111 & & & .128 \\
\hline Yes & 31 & 33 & & 19 & 45 & & 8 & 56 & \\
\hline No & 164 & 189 & & 142 & 211 & & 73 & 280 & \\
\hline
\end{tabular}

Age: mean \pm standard deviation, median, and range, $59.9 \pm 9.13$ years, 60 , and 36-82, respectively. CSF-1, Colony-stimulating factor-1; IL-6, interleukin-6; SCC, squamous cell carcinoma; $A d S q$, adenosquamous carcinoma; $T N M$, tumor node metastasis.

\section{Immunohistochemistry Staining of Tumor Samples and Association Between CSF-1, IL-6, and CD68 Expression and Clinicopathologic Variables}

Positive staining of CSF-1 and IL- 6 was mainly present on the cytoplasm of the tumor cells. Most of the alveolar epithelium and stromal cells were negative, although sporadic positive staining was seen on inflammatory cells, such as lymphocytes, macrophages, or plasmocytes (Figure 1, A-H). CD68 stained mostly in the cytoplasm of the tumor interstitial macrophages but not on the tumor cells, lymphocytes, plasmocytes, or fibroblasts (Figure 1, $I-L$ ). The positive expression rate of CSF-1, IL-6, and CD68 was $53.2 \%, 61.4 \%$, and $80.6 \%$, respectively. Also, of the 417 patients with NSCLC, $114(27.3 \%)$ were both
CSF-1-negative (CSF-1-) and IL-6-negative (IL-6-), and $175(42.0 \%)$ were both CSF-1-positive (CSF-1+) and IL-6-positive (IL-6+). The CSF-1, IL-6, and CD68 expression levels in the whole tumor sections were completely consistent with the results from the TMA.

To elucidate the biologic significance, we investigated the association of the clinicopathologic features and CSF1, IL-6, and CD68 expression levels. Significant differences in the immunohistochemical staining of CSF-1 and IL-6 were present among stage I, II, and III $(P=.030$ and $P=.010$, respectively), subcarinal lymph node status $(P=.028$ and $P<.001$, respectively), the number of involved nodes $(P=.020$ and $P=.017$, respectively), and number of involved nodal stations $(P=.004$ and 
TABLE 2. Clinicopathologic patient characteristics and univariate survival analysis

\begin{tabular}{|c|c|c|c|c|c|}
\hline Variable & $\begin{array}{l}\text { Patients } \\
\text { (n) }\end{array}$ & $\begin{array}{c}5-y \mathrm{OS} \\
\text { rate }(\%)\end{array}$ & $\begin{array}{c}P \\
\text { value }\end{array}$ & $\begin{array}{l}\text { 5-y DFS } \\
\text { rate }(\%)\end{array}$ & $\begin{array}{c}P \\
\text { value }\end{array}$ \\
\hline Gender & & & .945 & & .881 \\
\hline Male & $231(55.4)$ & 42.6 & & 40.3 & \\
\hline Female & $186(44.6)$ & 41.9 & & 40.1 & \\
\hline Age & & & .714 & & .879 \\
\hline$>60 \mathrm{y}$ & $198(47.5)$ & 42.0 & & 40.0 & \\
\hline$\leq 60 \mathrm{y}$ & $219(52.5)$ & 42.2 & & 40.7 & \\
\hline Smoking status & & & .211 & & .136 \\
\hline$\leq 40$ pack-y & $220(52.8)$ & 43.5 & & 41.6 & \\
\hline$>40$ pack-y & $197(47.2)$ & 41.0 & & 38.6 & \\
\hline Resection type & & & .113 & & .057 \\
\hline Pneumonectomy & $38(9.1)$ & 21.5 & & 19.9 & \\
\hline Lobectomy & 347 (83.2) & 45.7 & & 43.9 & \\
\hline $\begin{array}{l}\text { Bronchial sleeve } \\
\text { resection }\end{array}$ & $32(7.7)$ & 41.0 & & 37.9 & \\
\hline TNM stage & & & $<.001$ & & $<.001$ \\
\hline I & $162(38.8)$ & 69.2 & & 62.2 & \\
\hline II & $103(24.7)$ & 41.6 & & 38.9 & \\
\hline III & $152(36.5)$ & 19.3 & & 16.2 & \\
\hline Histologic subtype & & & .111 & & .063 \\
\hline Adenocarcinoma & $312(74.8)$ & 41.3 & & 40.1 & \\
\hline $\mathrm{SCC}$ & $102(24.5)$ & 44.0 & & 41.9 & \\
\hline AdSq & $3(0.7)$ & 0 & & 0 & \\
\hline $\begin{array}{l}\text { Subcarinal lymph node } \\
\text { status }\end{array}$ & & & $<.001$ & & $<.001$ \\
\hline Negative & $336(80.6)$ & 48.0 & & 46.8 & \\
\hline Positive & $81(19.4)$ & 15.1 & & 11.6 & \\
\hline Involved nodes (n) & & & $<.001$ & & $<.001$ \\
\hline$<4$ & 307 (73.6) & 51.0 & & 49.0 & \\
\hline$\geq 4$ & $110(26.4)$ & 18.4 & & 15.9 & \\
\hline Involved nodal stations (n) & & & $<.001$ & & $<.001$ \\
\hline$\leq 1$ & $285(68.3)$ & 53.8 & & 51.9 & \\
\hline$>1$ & $132(31.7)$ & 17.5 & & 14.5 & \\
\hline Adjuvant chemotherapy & & & .122 & & .075 \\
\hline Yes & $274(65.7)$ & 37.9 & & 35.4 & \\
\hline No & $143(34.3)$ & 42.6 & & 40.1 & \\
\hline Adjuvant radiotherapy & & & .077 & & .056 \\
\hline Yes & $64(15.4)$ & 39.2 & & 35.4 & \\
\hline No & $353(84.6)$ & 46.3 & & 44.5 & \\
\hline CSF-1 & & & $<.001$ & & $<.001$ \\
\hline Negative & 195 (46.7) & 59.6 & & 58.2 & \\
\hline Positive & $222(53.2)$ & 28.5 & & 25.7 & \\
\hline IL-6 & & & $<.001$ & & $<.001$ \\
\hline Negative & $161(38.6)$ & 59.6 & & 59.4 & \\
\hline Positive & $256(61.4)$ & 32.2 & & 28.6 & \\
\hline CD68 & & & .187 & & .138 \\
\hline Negative & $81(19.4)$ & 47.6 & & 45.8 & \\
\hline Positive & $336(80.6)$ & 41.0 & & 38.9 & \\
\hline CSF-1 plus IL-6 & & & $<.001$ & & $<.001$ \\
\hline CSF-1-, IL6- & $114(27.3)$ & 64.0 & & 63.2 & \\
\hline $\begin{array}{l}\text { CSF-1+, IL-6- and } \\
\text { CSF-1-, IL-6+ }\end{array}$ & $128(30.7)$ & 51.1 & & 50.1 & \\
\hline CSF-1+, IL-6+ & $175(42.0)$ & 23.7 & & 19.9 & \\
\hline CSF-1 plus CD68 & & & $<.001$ & & $<.001$ \\
\hline
\end{tabular}

TABLE 2. Continued

\begin{tabular}{lccccc}
\hline \multicolumn{1}{c}{ Variable } & $\begin{array}{c}\text { Patients } \\
\text { (n) }\end{array}$ & $\begin{array}{c}\text { 5-y OS } \\
\text { rate (\%) }\end{array}$ & $\begin{array}{c}\boldsymbol{P} \\
\text { value }\end{array}$ & $\begin{array}{c}\text { 5-y DFS } \\
\text { rate (\%) }\end{array}$ & $\begin{array}{c}\boldsymbol{P} \\
\text { value }\end{array}$ \\
\hline CD68- & $81(19.4)$ & 47.0 & & 45.1 & \\
CD68+, CSF-1- & $141(33.8)$ & 63.5 & & 62.8 & \\
CD68+, CSF-1+ & $195(46.8)$ & 26.2 & & 23.3 & \\
IL-6 plus CD68 & & & $<.001$ & & $<.001$ \\
CD68- & $81(19.4)$ & 47.0 & & 45.1 & \\
CD68+, IL-6- & $115(27.6)$ & 64.3 & & 63.3 & \\
CD68+, IL-6+ & $221(53.0)$ & 30.3 & & 26.5 & \\
CD68 plus CSF-1, IL-6 & & & $<.001$ & & $<.001$ \\
CD68- & $81(19.4)$ & 47.6 & & 45.8 & \\
CD68+, CSF-1+, IL-6+ $154(36.9)$ & 20.3 & & 16.1 & \\
CD68+, CSF1-, IL-6- & $74(17.7)$ & 69.4 & & 69.4 & \\
CD68+, CSF-1+, IL6- & $41(9.9)$ & 50.7 & & 46.7 & \\
CD68+, CSF1-, IL-6+ & $67(16.1)$ & 51.7 & & 48.9 & \\
CD68+, CSF-1+, IL-6+ & & & .001 & & .001 \\
CD68-, CSF-1+, IL-6+ & $24(13.5)$ & 51.3 & & 49.3 & \\
CD68+, CSF-1+, IL-6+ 154(86.5) & 20.3 & & 16.1 & \\
\hline
\end{tabular}

Data in parentheses are percentages. $O S$, Overall survival; $D F S$, disease-free survival; $S C C$, squamous cell carcinoma; $A d S q$, adenosquamous carcinoma; $C S F-1$, colonystimulating factor-1; $I L-6$, interleukin-6; - , negative; + , positive; $T N M$, tumor node metastasis.

$P=.010$, respectively; Table 1). Nevertheless, an analysis between the TAMs and clinicopathologic variables revealed no significant associations. To clarify the correlation between CSF-1 or IL-6 and TAMs, the nonparametric Spearman test was used for the correlation analysis. The results showed that the expression level of CSF-1 and IL-6 in the tumor specimens correlated positively with the infiltration degree of TAMs in the tumor stromal cells $(r=0.184$ and $r=0.196$, respectively; $P<.001$ ).

\section{Univariate and Multivariate Analyses for OS and DFS}

The 5-year OS and DFS rate was $36.8 \%$ and $31.7 \%$ for the total study population, respectively. Univariate analyses revealed that postoperative stage, subcarinal lymph node status, number of involved nodes, and number of involved nodal station were significant prognostic factors for survival $(P<.001$; Table 2$)$. The 5 -year OS and DFS rates in the CSF-1+ group were significantly lower than those in the CSF-1 - group $(28.5 \%$ vs $59.6 \%, P<.001 ; 25.7 \%$ vs $58.2 \%, P<.001)$. The IL-6- group had significantly greater 5-year OS $(59.6 \%$ vs $32.2 \%, P<.001)$ and DFS $(59.4 \%$ vs $28.6 \%, P<.001)$ rates than those of the IL$6+$ group. The survival data also showed that the 5-year OS $(64.0 \%)$ and DFS $(63.2 \%)$ rates for the CSF-1- and IL-6- group were better than those for the CSF-1+ or IL$6+$ group $(51.1 \%$ and $50.1 \%)$ and were better than those for the CSF-1+ and IL-6+ group (23.7\% and 19.9\%; $P<.001)$. However, no significant difference was found between the CD68-positive (CD68+) group and the CD68negative (CD68-) group for both OS $(41.0 \%$ vs $47.6 \%$, $P=.187$; Figure $2, A)$ and DFS $(38.9 \%$ vs $45.8 \%$, 

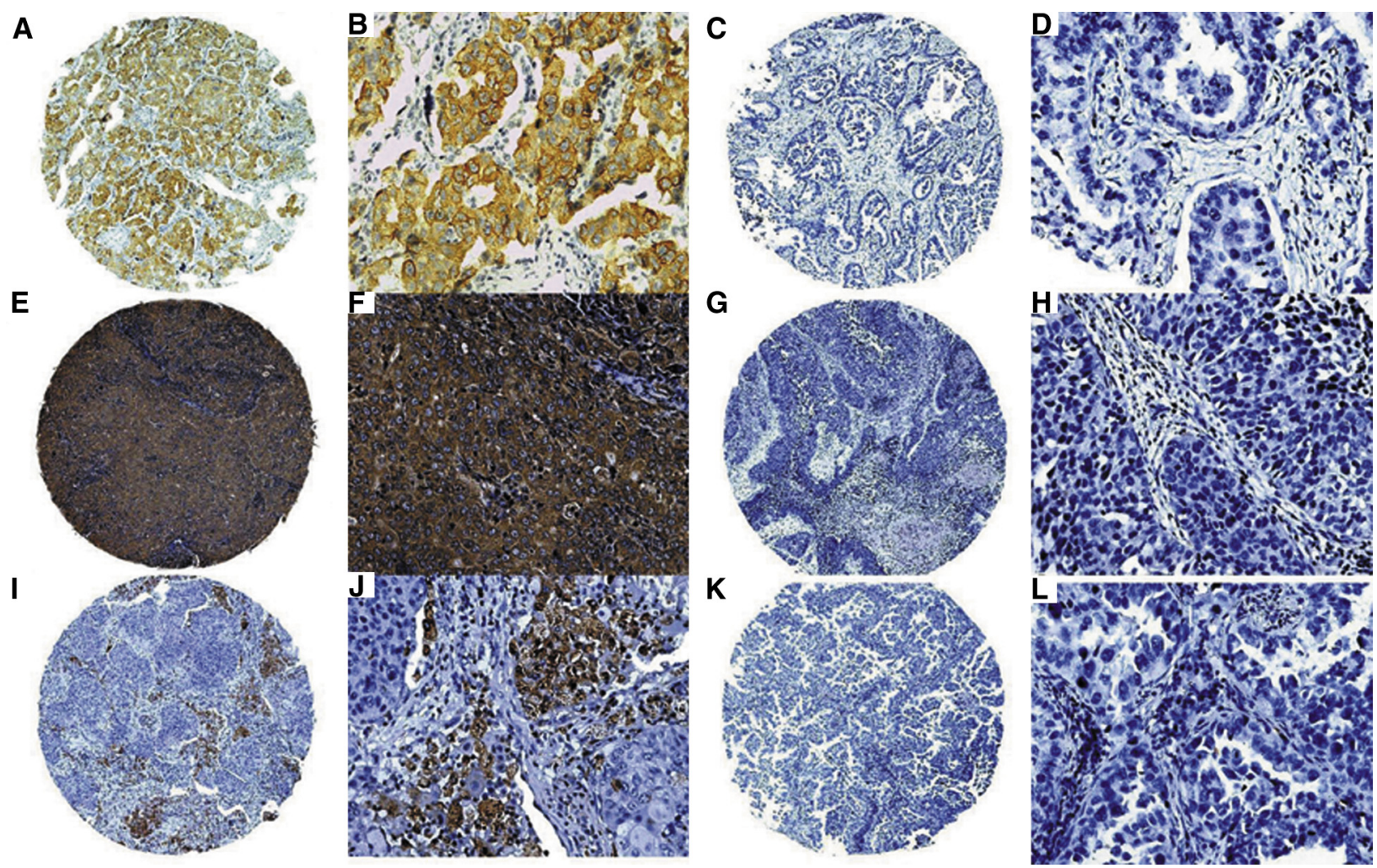

FIGURE 1. Typical expression images of macrophage colony-stimulating factor (CSF-1), interleukin-6 (IL-6), and CD68 immunohistochemistry in tissue microarrays. Original magnification: CSF-1-positive (CSF1+): A, $\times 40$; and B, $\times 200$; CSF-1-negative (CSF1-): C, $\times 40$; and D, $\times 200$; IL-6-positive (IL6+): E, $\times 40$; and F, $\times 200$; IL-6-negative (IL6-): G, ×40; H, ×200; CD68-positive (CD68+): I, $\times 40$ and J, ×200; CD68-negative (CD68-): K, $\times 40$; and $\mathrm{L}, \times 200 \times$.

$P=.138$; Figure $2, B)$. Additional analysis proved that the 5 -year OS $(20.3 \%)$ and DFS $(16.1 \%)$ rates in the group positive for CD68, CSF-1, and IL-6 were the worst, and the prognosis of the CD68+, CSF-1-, and IL-6- was the best $(69.4 \%$ and $69.4 \%$, respectively). The 5 -year OS $(50.7 \%)$ and DFS $(46.7 \%)$ rates for the CD68+, CSF$1+$, and IL-6- group and the 5-year OS $(51.7 \%)$ and DFS $(48.9 \%)$ rates for the CD68+, CSF-1-, and IL-6+ group were in between the group with the worst and the group with best rates $(P<.001$; Figure $3, A$ and $B)$. The differences in the 5-year OS and DFS rates between the CD68+, CSF-1-, IL-6- group and CD68+, CSF-1+, IL$6-$ group were statistically significant $(P=.015$ and $P=.017$, respectively; Figure 2, $C$ and $D$ ). A similar statistically significant difference was found between the CD68+, CSF-1-, IL-6- group and CD68+, IL-6+, CSF$1-(P=.007$ and $P=.002$, respectively; Figure 2, $E$ and $F)$.

Multivariate analysis determined that the postoperative stage, number of involved nodal stations, and coexpression of CSF-1, IL-6, and CD68 (OS hazard ratio [HR], 2.790; DFS HR, 3.063) remained the most significant and independent prognostic factors for survival $(P<.05)$. However, the combinations of CSF-1 and IL-6 (OS HR, 1.160; DFS HR, 1.153), CD68 and CSF-1 (OS HR, 1.180;
DFS HR, 1.163), and CD68 and IL-6 (OS HR, 1.047; DFS HR, 1.118), number of involved nodes, and subcarinal lymph node status were not $(P>.05$, Table 3$)$. To clarify whether TAMs played a major role in the prognosis, the survival data showed that 5 -year OS rate $(51.3 \%)$ and DFS rate $(49.3 \%)$ of the CD68-, CSF-1-, and IL-6+ group were better than those for the group with all 3 positive (CD68, CSF-1, IL-6; $19.6 \%$ and $15.7 \% ; P=.001$ and $P=.001$, respectively; Figure 3, $C$ and $D$ ). Based on the 5-year OS and DFS rates for the combination of CSF-1 and/or IL-6 and TAM, we performed a stratified analysis according to stage and histologic type (Table E1 and Figure E1). The TAM, CSF-1, IL-6 phenotype remained a valuable predictor for the specific stage and uniformly histologic type.

\section{DISCUSSION}

It is well known that macrophages are a fundamental part of the innate defense mechanisms and can promote specific immunity by inducing T-cell recruitment and activation. Despite this, accumulating evidence has shown that their presence within the tumor microenvironment is associated with enhanced tumor progression through production of angiogenic factors and immunosuppression. ${ }^{19,20}$ However, 

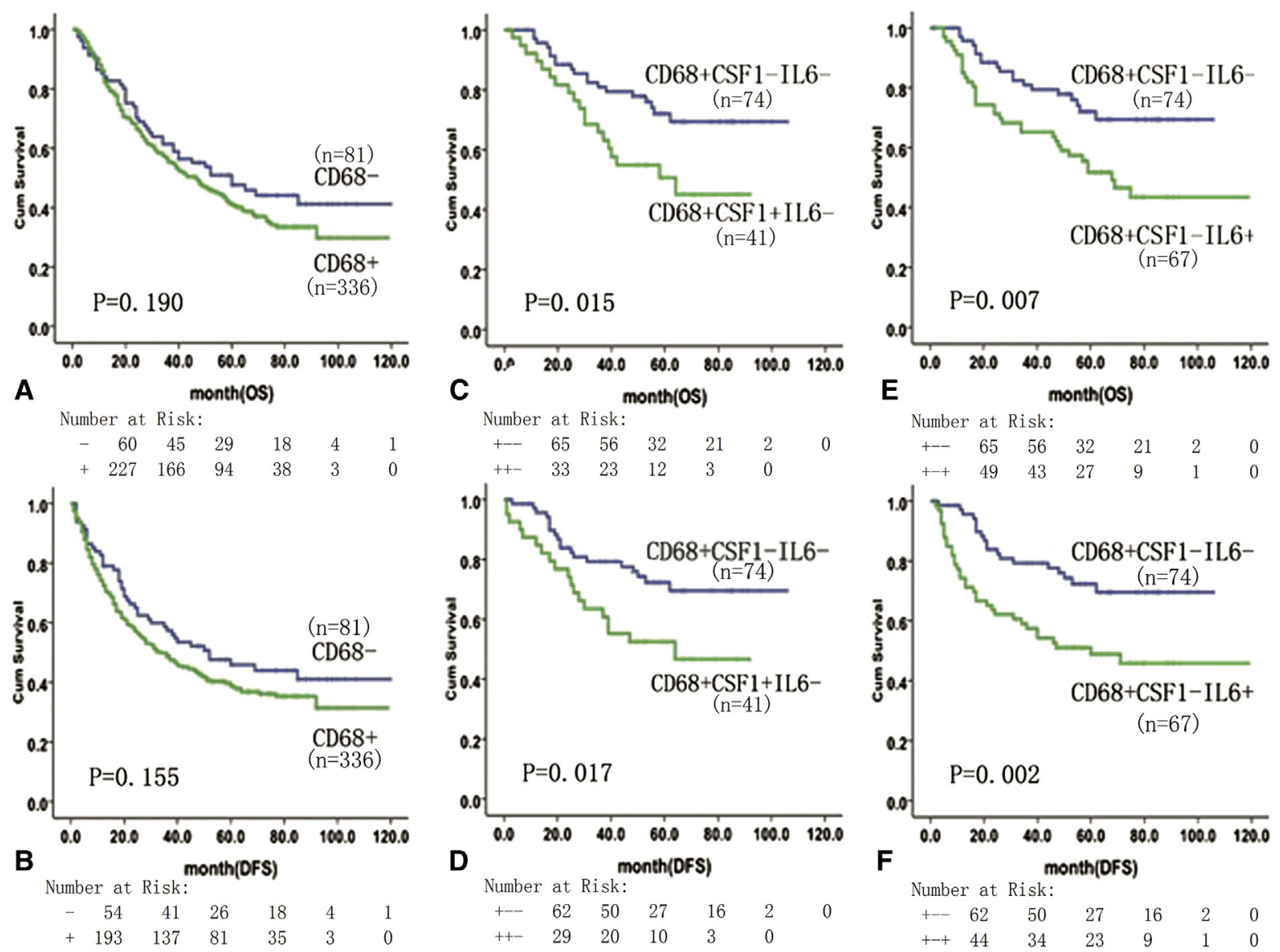

FIGURE 2. Prognostic significance assessed using Kaplan-Meier survival estimates and log-rank tests. Kaplan-Meier analysis of (A) overall survival (OS) and (B) disease-free survival (DFS) for CD68. Comparisons of OS and DFS by (C) CD68-positive (CD68+), colony-stimulating factor-1 (CSF-1)-positive (CSF1+), interleukin (IL)-6-negative (IL6-) and (D) CD68+, CSF-1-negative (CSF1-), IL-6-negative (IL6-); (E) CD68+, CSF1-, IL6+ and F, CD68+, CSF1-, IL6-. Cum, Cumulative.

studies with clinical samples to date have shown contradictory results for the prediction of the prognosis in patients with NSCLC. ${ }^{9-12,21}$ In certain cases, infiltration of TAMs correlated with a poor prognosis in patients with NSCLC. ${ }^{10,21}$ Nevertheless, our results showed that interstitial TAMs were not significantly associated with prognosis $(P>.05)$, which was partly consistent with other overseas studies. ${ }^{11}$ Several studies have even found that the infiltration of TAMs was related to a good prognosis in patients with NSCLC. ${ }^{12}$ This paradoxical role of TAMs can be explained by their functional plasticity, which might result in the polarized expression of either pro- or antitumor functions.

TAMs are derived from circulating monocytes that are recruited into tumors by a range of growth factors and chemokines, such as CSF-1, IL-6, and a series of chemotactic cytokines, and these cytokines are mainly produced by tumor cells. ${ }^{19,22-24}$ The present study analyzed the expression levels of CSF-1 and IL-6 using immunohistochemistry to clarify whether co-expression of CSF-1 and
IL-6 was associated with interstitial TAMs. CSF-1 is a hematopoietic growth factor that stimulates the proliferation and differentiation of monocytes to macrophages. ${ }^{25} \mathrm{CSF}-$ 1 plays an important role in the physiology of several cancers, and its expression has been correlated with tumor cell invasiveness and poor patient prognosis. ${ }^{26,27}$ IL- 6 is one of the major immune-regulatory cytokines present in the tumor microenvironment and induces several pathways, leading to tumor proliferation, angiogenesis, and chemotherapy resistance. ${ }^{28,29}$ In addition, the pre- and postoperative serum levels of CSF-1 and IL-6 were important prognostic indicators in patients with $\mathrm{NSCLC}^{16,17}$; however, few histologic investigations have been reported.

In the present study, univariate analysis for survival proved that the combination of CD68, CSF-1, and IL-6 was an important prognostic factor $(P<.001)$. The 5 -year OS and DFS rates in the group with all 3 positive (CD68, CSF-1, IL-6) were the worst. The prognosis of the CD68+, CSF-1-, IL-6- group was the best. As shown in Figure 2, the 5-year OS and DFS rates in the CD68+, 



FIGURE 3. Kaplan-Meier analysis of (A) overall survival $(O S)$ and (B) disease-free survival (DFS) for the combination of CD68, colony-stimulating factor-1 (CSF-1), and interleukin-6 (IL-6). Comparisons of OS and DFS by (C) CD68-negative (CD68-), CSF-1-positive (CSF1+), IL-6-positive (IL6+) and (D) CD68-positive (CD68+), CSF1+, IL6+. Cum, Cumulative.

CSF-1 - IL-6 - group were better than those in the CD68+, CSF-1+, IL-6- group or the CD68+, CSF-1-, IL-6+ group $(P<.05)$. In addition, correlation analysis showed that the expression level of CSF-1 and IL-6 in tumor correlated positively with the infiltration degree of TAMs in the tumor stromal cells $(P<.001)$. From on these results, it was assumed that TAMs were recruited into tumor sites by tumor-derived CSF-1 and IL-6 and educated toward a tumor-promoting phenotype; however, TAMs that were recruited into tumor sites by other cytokines, such as IL-12, IL-23, tumor necrosis factor- $\alpha$, and interferon- $\gamma$, might continue to have tumor-suppressing functions. ${ }^{30}$ This is probably why the infiltration of TAMs has been controversial in the prognosis of patients with NSCLC. These findings have been confirmed by several experimental results worldwide. ${ }^{13,30-32}$ For instance, Kawamura and colleauges ${ }^{13}$ suggested that CSF-1 derived from tumor tissues would induce TAMs to shift toward the M2 phenotype, which was considered to promote tumor growth. Dijkgraaf and colleagues ${ }^{31}$ demonstrated that most cervical and ovarian cancer cells either hampered monocyte to dendritic cell differentiation or skewed their differentiation toward M2-like TAMs, depending on their ability to produce prostaglandin $\mathrm{E}_{2}$ and IL-6. Blocking these cytokines completely restored their differentiation toward dendritic cells. ${ }^{31}$

Furthermore, our data have suggested that the combination of CSF-1 and IL-6 had a stronger ability to induce tumor-promoting TAMs. As shown by univariate analysis, no significantly different prognosis was found between the CD68+, CSF-1-, IL-6+ group or the CD68+, CSF$1+$, IL-6- group and the CD68 - group, although a statistically significant difference was present between the CD68+, CSF-1-, IL-6+ group or the CD68+, CSF-1+, IL-6- group and the CD68+, CSF-1+, IL-6+ group (Figure 3, $A$ and $B$ ). These results were consistent with several reports. ${ }^{14,31,33,34}$ For example, Duluc and colleagues ${ }^{14}$ found that tumor-associated leukemia inhibitory factor and IL-6 skew monocyte differentiation into tumor-promoting TAMs by enabling monocytes to consume CSF-1. Also, Yang and colleagues ${ }^{33}$ certified that CSF-1 cooperating with NF-kB induces TAMs into the tumorpromoting phenotype by upregulating c-Jun. In addition, Dijkgraaf and colleagues ${ }^{31}$ and Heusinkveld and colleagues $^{34}$ reported that prostaglandin $\mathrm{E}_{2}$ and IL-6 were associated with chemotherapy resistance and the tumorinduced differentiation of tumor-promoting macrophages. Accordingly, it is likely that tumor-promoting TAM 
TABLE 3. Multivariate analysis of factors associated with overall survival and disease-free survival

\begin{tabular}{lcr}
\hline \multicolumn{1}{c}{ Variable } & HR (95\% CI) & $\boldsymbol{P}$ value \\
\hline OS & & \\
Involved nodes (n $\geq 4$ vs $<4)$ & $1.085(0.686-1.717)$ & .728 \\
Involved nodal stations (n >1 vs $\leq 1)$ & $1.538(1.060-2.232)$ & .024 \\
TNM (stage II and III vs stage I) & $1.590(1.273-1.986)$ & $<.001$ \\
Subcarinal lymph node status (- vs +) & $1.047(0.717-1.530)$ & .810 \\
CSF-1+, IL-6+ vs CSF-1-, IL-6- and & $1.160(0.616-2.182)$ & .646 \\
$\quad$ CSF-1+ or IL-6+ & & \\
CD68+, CSF-1+ vs CD68+, CSF-1- & $1.180(0.693-2.011)$ & .542 \\
$\quad$ and CD68- & & \\
CD68+, IL-6+ vs CD68+, IL-6- and & $1.047(0.660-1.660)$ & .847 \\
$\quad$ CD68- & & \\
CD68+, CSF-1+, IL-6+ vs other* & $2.790(2.158-3.607)$ & $<.001$ \\
DFS & & \\
Involved nodes (n $\geq 4$ vs $<4)$ & $1.132(0.725-1.767)$ & .587 \\
Involved nodal stations (>1 vs $\leq 1)$ & $1.656(1.142-2.402)$ & .008 \\
TNM (stage I and III vs stage I) & $1.568(1.257-1.955)$ & $<.001$ \\
Subcarinal lymph node (- vs +) & $1.281(0.880-1.866)$ & .196 \\
CSF-1+, IL-6+ vs CSF-1-, IL-6- and & $1.153(0.612-2.171)$ & .660 \\
$\quad$ CSF-1+ or IL-6+ & & \\
CD68+, CSF-1+ vs CD68+, CSF-1- & $1.163(0.683-1.981)$ & .579 \\
$\quad$ and CD68- & & \\
CD68+, IL-6+ vs CD68+, IL-6- and & $1.118(0.704-1.774)$ & .636 \\
$\quad$ CD68- & & \\
CD68+, CSF-1+, IL-6+ vs other* & $3.063(2.364-3.968)$ & $<.001$ \\
\hline
\end{tabular}

$H R$, Hazard ratio; $C I$, confidence interval; $O S$, overall survival; $D F S$, disease-free survival; $C S F-1$, colony-stimulating factor- $1 ;+$, positive; $I L-6$, interleukin- 6 ; - , negative; TNM, tumor node metastasis. *Included CD68+, CSF-1+, IL6-; CD68+, CSF-1-, IL-6+; CD68+, CSF-1-, IL-6-; and CD68-.

generation requires the co-expression of CSF-1 and IL- 6 or other additional cytokines to participate jointly.

To clarify whether TAMs played a major role in the prognosis, we compared the prognostic differences between the CD68-, CSF-1+, IL-6+ group and the CD68+, CSF-1+, IL-6+ group, and the HRs between the combinations of CSF-1 and IL-6, CD68 and CSF-1, CD68 and IL-6, and CD68, CSF-1, and IL-6. Figure 3, $C$ and $D$, showed that the 5-year OS and DFS rates for the CD68-, CSF-1+, IL-6+ group were better than those for the group with all 3 positive (CD68, CSF-1, IL-6; $P=.001$ and $P=.001$, respectively). Furthermore, in the multivariate analyses, the combination of CD68, CSF-1, and IL-6 (OS HR, 2.790; DFS HR, 3.063) remained the most significant and independent prognostic factor for survival in the patients with NSCLC $(P<.05)$, rather than the combination of CSF-1 and IL-6 (OS HR, 1.160; DFS HR, 1.153), CD68 and CSF-1 (OS HR, 1.180; DFS HR, 1.163), or CD68 and IL-6 (OS HR, 1.047; DFS HR, 1.118; $P>$.05). However, a few flaws are present in the correlative data, such as the semiquantitative assessment and the relatively small sample sizes. To some extent, these results indicated that CSF-1 and IL-6 indirectly influenced the prognosis of patients with NSCLC through the TAMs. Accumulating evidence has supported the hypothesis that the effects on TAMs might contribute to the antitumor effect of bisphosphonates; thus, perhaps, TAMs could be regarded as potential targets of bisphosphonates. ${ }^{35}$

Approximately $40 \%$ of patients with stage I NSCLC will die within 5 years after curative pulmonary resection, mainly from the development of distant metastases and local recurrence. ${ }^{4-6}$ Classifying those patients in stage I who have a greater risk of recurrence or metastasis is necessary to providing timely measures to avoid it. From the 5-year OS and DFS rates for the combination of CSF1 and IL-6 and TAMs, we performed a stratified analysis according to stage and histologic type. The TAM-positive, CSF-1+, IL-6+ phenotype remained a valuable predictor for the specific stage and uniformly histologic type. Thus, undertaking adjuvant treatment early in patients with TAM, CSF-1, and IL-6 positive after surgery for stage I adenocarcinoma might reduce tumor recurrence and prolong survival.

\section{CONCLUSIONS}

The combination of CD68, CSF-1, and IL-6 was a significant and independent prognostic factor for patients with NSCLC undergoing curative resection. As possible messengers, CSF-1 and IL-6 might be involved in the interaction between cancer cells and TAMs and play a pivotal role in TAMs' malignant phenotype transition. However, the detailed signaling pathway and underlying regulatory mechanisms still need to be investigated further in-depth.

\section{References}

1. Siegel R, Naishadham D, Jemal A. Cancer statistics, 2013. CA Cancer J Clin. 2013;63:11-30.

2. Fahham D, Weiss ID, Abraham M, Beider K, Hanna W, Shlomez Z, et al. In vitro and in vivo therapeutic efficacy of CXCR4 antagonist BKT140 against human non-small cell lung cancer. J Thorac Cardiovasc Surg. 2012;144:1167-75.

3. Miyoshi K, Moriyama S, Kunitomo T, Nawa S. Prognostic impact of intratumoral vessel invasion in completely resected pathologic stage I non-small cell lung cancer. J Thorac Cardiovasc Surg. 2009;137:429-34.

4. Reck M, Heigener DF, Mok T, Soria JC, Rabe KF. Management of non-smallcell lung cancer: recent developments. Lancet. 2013;382:709-19.

5. Varlotto JM, Recht A, Flickinger JC, Medford-Davis LN, Dyer AM, Decamp MM. Factors associated with local and distant recurrence and survival in patients with resected nonsmall cell lung cancer. Cancer. 2009;115:1059-69.

6. Rinewalt D, Shersher DD, Daly S, Fheid C, Basu S, Mahon B, et al. Development of a serum biomarker panel predicting recurrence in stage I non-small cell lung cancer patients. J Thorac Cardiovasc Surg. 2012;144:1344-50.

7. Hagemann T, Wilson J, Burke F, Kulbe H, Li NF, Plüddemann A, et al. Ovarian cancer cells polarize macrophages toward a tumor-associated phenotype. J Immunol. 2006;176:5023-32.

8. Balkwill F, Charles KA, Mantovani A. Smoldering and polarized inflammation in the initiation and promotion of malignant disease. Cancer Cell. 2005;7:211-7.

9. Becker M, Müller CB, De Bastiani MA, Klamt F. The prognostic impact of tumor-associated macrophages and intra-tumoral apoptosis in non-small cell lung cancer. Histol Histopathol. 2014;29:21-31.

10. da Costa Souza P, Parra ER, Atanazio MJ, da Silva OB, Noleto GS, Ab'saber AM, et al. Different morphology, stage and treatment affect immune cell infiltration and long-term outcome in patients with non-small-cell lung carcinoma. Histopathology. 2012;61:587-96.

11. Kim DW, Min HS, Lee KH, Kim YJ, Oh DY, Jeon YK, et al. High tumour islet macrophage infiltration correlates with improved patient survival but not with 
EGFR mutations, gene copy number or protein expression in resected non-small cell lung cancer. Br J Cancer. 2008;98:1118-24.

12. Welsh TJ, Green RH, Richardson D, Waller DA, O'Byrne KJ, Bradding P. Macrophage and mast-cell invasion of tumor cell islets confers a marked survival advantage in non-small-cell lung cancer. J Clin Oncol. 2005;23:8959-67.

13. Kawamura K, Komohara Y, Takaishi K, Katabuchi H, Takeya M. Detection of M2 macrophages and colony-stimulating factor 1 expression in serous and mucinous ovarian epithelial tumors. Pathol Int. 2009;59:300-5.

14. Duluc D, Delneste Y, Tan F, Moles MP, Grimaud L, Lenoir J, et al. Tumor-associated leukemia inhibitory factor and IL-6 skew monocyte differentiation into tumor-associated macrophage-like cells. Blood. 2007;110:4319-30.

15. Sica A, Larghi P, Mancino A, Rubino L, Porta A, Totaro MG, et al. Macrophage polarization in tumour progression. Semin Cancer Biol. 2008;18:349-55.

16. Kaminska J, Kowalska M, Kotowicz B, Fuksiewicz M, Glogowski M, Wojcik E, et al. Pretreatment serum levels of cytokines and cytokine receptors in patients with non-small cell lung cancer, and correlations with clinicopathological features and prognosis. M-CSF-an independent prognostic factor. Oncology. 2006;70:115-25.

17. Kita H, Shiraishi Y, Watanabe K, Suda K, Ohtsuka K, Koshiishi Y, et al. Does postoperative serum interleukin-6 influence early recurrence after curative pulmonary resection of lung cancer? Ann Thorac Cardiovasc Surg. 2011;17:454-60.

18. Salgado RA, Snoeckx A, Spinhoven M, Op de Beeck B, Corthouts B, Parizel PM. Update in non small-cell lung cancer staging. JBR-BTR. 2013;96:118-22.

19. Pollard JW. Tumour-educated macrophages promote tumour progression and metastasis. Nat Rev Cancer. 2004;4:71-8.

20. Wang R, Zhang J, Chen S, Lu M, Luo X, Yao S, et al. Tumor-associated macrophages provide a suitable microenvironment for non-small lung cancer invasion and progression. Lung Cancer. 2011;74:188-96.

21. Chen JJ, Yao PL, Yuan A, Hong TM, Shun CT, Kuo ML, et al. Up-regulation of tumor interleukin-8 expression by infiltrating macrophages: its correlation with tumor angiogenesis and patient survival in non-small cell lung cancer. Clin Cancer Res. 2003;9:729-37.

22. Sica A, Allavena P, Mantovani A. Cancer related inflammation: the macrophage connection. Cancer Lett. 2008;267:204-15.
23. Lucas T, Abraham D, Aharinejad S. Modulation of tumor associated macrophages in solid tumors. Front Biosci. 2008;13:5580-8.

24. Sica A, Bronte V. Altered macrophage differentiation and immune dysfunction in tumor development. J Clin Invest. 2007;117:1155-66.

25. Kascinski B. Expression of CSF-1 and its receptor CSF-1R in non-hematopoietic neoplasms. Cancer Treat Res. 2002;107:285-92.

26. Jones C, Ricardo S. Macrophages and CSF-1: implications for development and beyond. Organogenesis. 2013;9:249-60.

27. Curry JM, Eubank TD, Roberts RD, Wang Y, Pore N, Maity A, et al. M-CSF signals through the MAPK/ERK pathway via Sp1 to induce VEGF production and induces angiogenesis in vivo. PLoS One. 2008;3:e3405.

28. Hodge DR, Hurt EM, Farrar WL. The role of IL-6 and STAT3 in inflammation and cancer. Eur J Cancer. 2005;41:2502-12.

29. Dijkgraaf EM, Welters MJ, Nortier JW, van der Burg SH, Kroep JR. Interleukin6/IL-6 receptor pathway as a new therapy target in epithelial ovarian cancer. Curr Pharm Des. 2012;18:3816-27.

30. Lippitz BE. Cytokine patterns in patients with cancer: a systematic review. Lancet Oncol. 2013;14:e218-28.

31. Dijkgraaf EM, Heusinkveld M, Tummers B, Vogelpoel LT, Goedemans R, Jha V, et al. Chemotherapy alters monocyte differentiation to favor generation of cancer-supporting M2 macrophages in the tumor micro-environment. Cancer Res. 2013;73:2480-92.

32. Goswami S, Sahai E, Wyckoff JB, Cammer M, Cox D, Pixley FJ, et al. Macrophages promote the invasion of breast carcinoma cells via a colony-stimulating factor-1/epidermal growth factor paracrine loop. Cancer Res. 2005;65:5278-83.

33. Yang Y, Qin J, Lan L, Li N, Wang C, He P, et al. M-CSF cooperating with NF-kB induces macrophage transformation from M1 to M2 by up-regulating c-Jun. Cancer Biol Ther. 2014;15:99-107.

34. Heusinkveld M, de Vos van Steenwijk PJ, Goedemans R, Ramwadhdoebe TH, Gorter A, Welters MJ, et al. M2 macrophages induced by prostaglandin $\mathrm{E}_{2}$ and IL-6 from cervical carcinoma are switched to activated M1 macrophages by CD4+ Th1 cells. J Immunol. 2011;187:1157-65.

35. Rogers TL, Holen I. Tumour macrophages as potential targets of bisphosphonates. J Transl Med. 2011;9:177. 

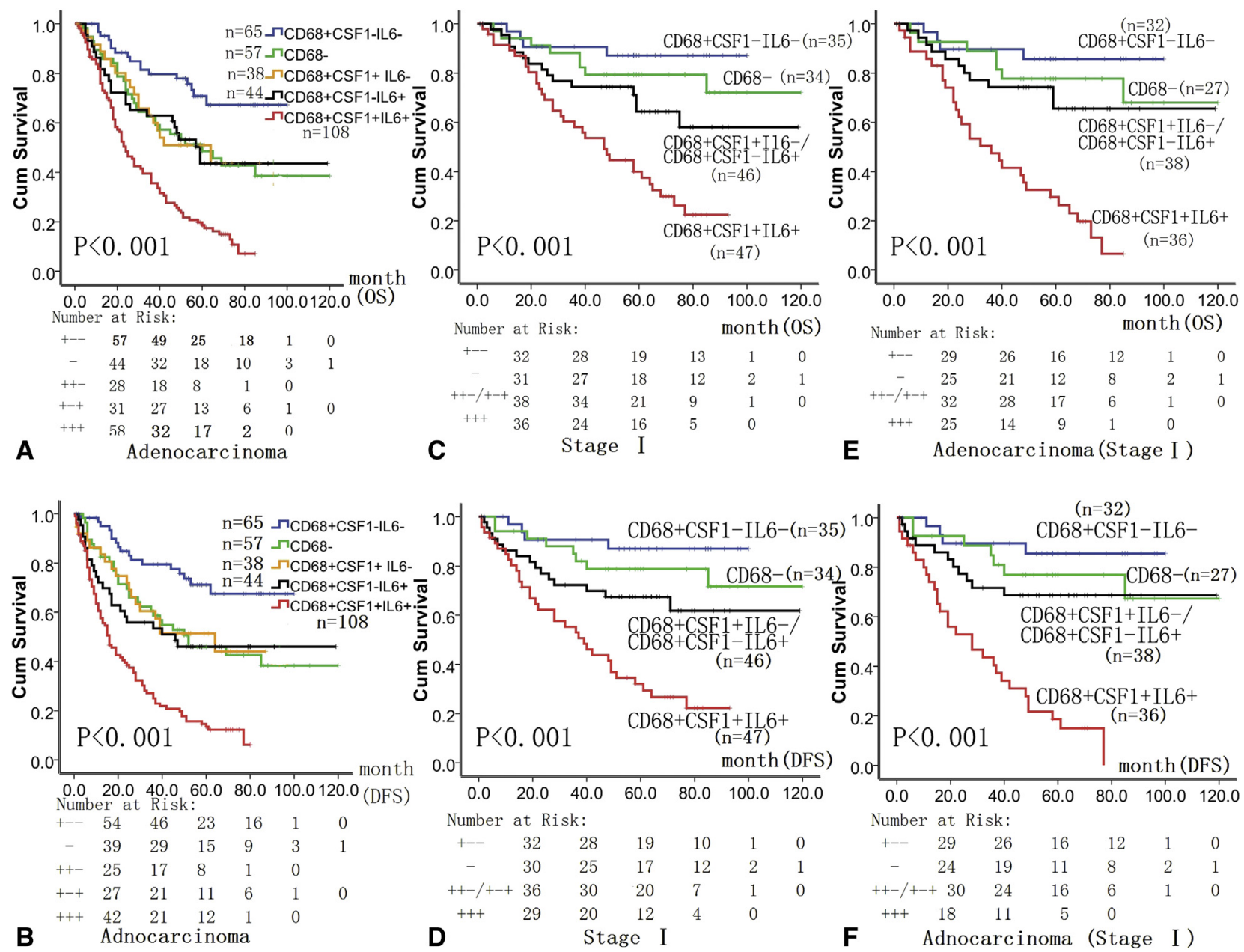

FIGURE E1. A and B, Overall survival $(O S)$ and disease-free survival $(D F S)$ for combined colony-stimulating factor (CSF-1) and interleukin-6 $(I L-6)$ and tumor-associated macrophages $(T A M s)$ in patients with adenocarcinoma. C and D, OS and DFS for combined CSF-1 and IL-6 and TAMs in patients with non-small cell lung cancer at stage I. E and F, OS and DFS for combined CSF-1 and IL-6 and TAMs in patients with stage I adenocarcinoma. Cum, Cumulative; +, positive; -, negative. 
TABLE E1. Five-year overall survival and disease-free survival of combination of CSF-1, IL-6, and CD68 stratified by stage and histologic type

\begin{tabular}{|c|c|c|c|c|c|}
\hline Variable & $\begin{array}{c}\text { CD68 }+, \\
\text { CSF-1 }+, \text { IL-6+ } \\
\end{array}$ & $\begin{array}{c}\text { CD68 }+, \text { CSF-1 }+, \text { IL-6- or } \\
\text { CD68 }+, \text { CSF-1 }-, \text { IL-6 }+\end{array}$ & $\begin{array}{c}\text { CD68+, CSF-1-, } \\
\text { IL-6- } \\
\end{array}$ & CD68- & $P$ value \\
\hline \multicolumn{6}{|c|}{ Overall survival } \\
\hline \multicolumn{6}{|c|}{ TNM stage } \\
\hline$I^{*}$ & 38.2 & 64.4 & 85.7 & 76.2 & $<.001$ \\
\hline $\mathrm{II}^{*}$ & 21.7 & 60.5 & 57.1 & 36.5 & .002 \\
\hline $\mathrm{III}^{*}$ & 5.5 & 24.1 & 45.9 & 16.0 & $<.001$ \\
\hline \multicolumn{6}{|c|}{ Histologic type } \\
\hline SCC* & 23.3 & 60.9 & 69.1 & 47.8 & .001 \\
\hline $\mathrm{AC}^{*}$ & 18.7 & $45.0 / 43.6$ & 68.5 & 48.6 & $<.001$ \\
\hline AdSq & 0.0 & 0.0 & & 0.0 & \\
\hline \multicolumn{6}{|l|}{ DFS } \\
\hline \multicolumn{6}{|l|}{ TNM stage } \\
\hline $\mathrm{I}^{*}$ & 30.3 & 64.4 & 85.7 & 75.3 & $<.001$ \\
\hline $\mathrm{II}^{*}$ & 21.7 & 58.2 & 52.5 & 30.5 & .001 \\
\hline III* & 1.7 & 18.3 & 43.0 & 14.1 & $<.001$ \\
\hline \multicolumn{6}{|c|}{ Histologic type } \\
\hline SCC* & 20.5 & 59.0 & 69.1 & 47.8 & .001 \\
\hline $\mathrm{AC}^{*}$ & 13.5 & $44.0 / 41.1$ & 68.3 & 44.3 & $<.001$ \\
\hline $\mathrm{AdSq}$ & 0.0 & 0.0 & & 0.0 & \\
\hline \multicolumn{6}{|l|}{ Stage I AC* } \\
\hline OS & 27.4 & 65.6 & 79.2 & 73.5 & $<.001$ \\
\hline DFS & 16.2 & 64.1 & 79.0 & 72.6 & $<.001$ \\
\hline
\end{tabular}

Data presented as percentages. $C S F-1$, Colony-stimulating factor-1; $I L-6$, interleukin- $6 ;+$, positive; -, negative; $S C C$, squamous cell carcinoma; $A C$, adenocarcinoma; $A d S q$, adenosquamous carcinoma; $T N M$, tumor node metastasis. ${ }^{*} P<.005$. 